\title{
Stress Analysis Near the Welding Interface Edges of a QFP Structure under Thermal Loading
}

\author{
Zhigang Huang, Xuecheng Ping, and Pingan Liu \\ School of Mechatronics Engineering, East China Jiaotong University, \\ Nanchang, Jiangxi Province, P.R. China, 330013 \\ Huangzg7301@163.com
}

\begin{abstract}
The purpose of this paper was to investigate the reason why the interface delaminations occurred in the QFP structure. The stress concentration was the key factor responsible for the microcrack occurrence. In order to figure out which factors affect the stress distribution, the material's attributes and the structure's dimension were considered. The finite element program ABAQUS6.9 together with the analytical solutions of singular stress fields was used to calculate the stresses near the interface edges. In conclusion, it appeared that strong mismatches of the thermal expansion coefficients and material elastic constants of the chip and PCB would lead to serious singular stresses at the interface edges. Stress intensity factor $K$ and stress singularity order $\lambda$ were given, based on which failure criterions could be established to predict the potential interface delamination. It is suggested that the good design of packaging structure depends on proper material selection and dimension determination.
\end{abstract}

Keywords: Electronic packaging, QFP structure, Interfacial thermal stresses, Stress concentration.

\section{Introduction}

Nowadays, quad flat packages(QFPs) are widely used in electronics engineering. A QFP structure often consists of metal pins, solder and PCBs. This kind of structure has two common features such as interfaces and corners. The solder layer plays an important role in the mechanical and electric connections. The require-ments of highdensity,cost-effective performance for package have resulted in very high IO count in smaller dimensions in the chip design(A.Q. Xu et al.2000)[1]. Due to the mismatch of thermal and mechanical properties in the dissimilar material structures as well as the change in working or environment temperature, high stress singularity will appear at the interface edges. Since the solder interlayers tend to be weak mechanically, in which microcracking or delamination are more easy to occur, the strength and service life of the whole structure will be directly reduced (Yi lan Kang 1996)[2]. Therefore, the awareness of the stress state near the interface edges is very essential to the strength evaluation and life prediction.

Many researchers have studied these problems and have brought some methods to relax singular stresses at the interface edges. Failure criterions have also been discussed by some scientists. The stress singularity problems at the free edges of bonded 
dissimilar materials were analyzed previously(Shuir 1989 and Munz and Yang 1992)[3,4]. The stress singularity problems of the edge of bonded bimaterial beams were discussed by some scholars (Kang et al. 1995)[5]. The stress singularities in an anisotropic wedge was analyzed (Chue et al.2001)[6]. The failure models and strength criterions were studied experimentally (Basaran et al. 2005 and Park et al. 2002)[7,8]. Effects of geometrical structure and dimension on thermal stress distribution were analyzed based on the finite element method (Jiang et al.2006 and Cho et al. 2009) $[9,10]$.

The failure usually initiates at the singular stress point, and the knowledge of interfacial fracture mechanics is important to understand the failure mechanisms and to achieve better structure design. Although some jobs have been done by some scholars in this area (Seiji Ioka,and Keiji Masuda, et al.1999)[13], there were relatively few papers to discuss the singular thermal stresses near the interface edges. It should be pointed out that, 1) the thermal stress is singular near the sharp corner of the electronic packages, it is difficult to calculate the singular stress fields by the conventional finite element method(J.M. Hu 1995)[11]; 2) the effects of solder thicknesses for a pin/PCB joint on the singular thermal stresses were not systematically studied. In this paper, the above two problems are going to be researched.

\section{Theoretical Background}

If two materials with different elastic properties are bonded together, stress singularities often occur at free edges of the interface under mechanical or thermal loading. A general configuration of a joint between two rectangular plates is shown in Fig.1. The stress states near the free edge of the interface (point o) is given by the following equation:

$$
\sigma_{i j}(r, \theta)=\frac{K}{(r / L)^{\lambda}} f_{i j}(\theta)+\sigma_{i j 0}(\theta)
$$

where $r$ and $\theta$ are the polar coordinates of the specified point o and $L$ is a characteristic length of the configuration. $\lambda$ is the stress singularity order, $f_{i j}(\theta)$ is the angular variations of stresses, and $K$ is the stress intensity factor. $\lambda, f_{i j}(\theta)$ and $K$ are all dependent on the material constants and the wedge angles or shapes. $\sigma_{i j 0}(\theta)$ is the

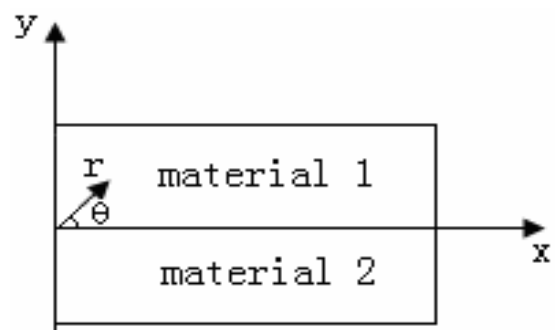

Fig. 1. A configuration of the interface edges 
constant stress term under the thermal loading. In order to determine its value, we can use the following formulas(Suga and Lancu 1989):

$$
\sigma_{\theta 0}=\frac{\sigma_{0}}{2}(1+\cos 2 \theta)
$$

if $\theta=0, \sigma_{\theta 0}=\sigma_{0}$. Where $\sigma_{0}$ is defined by:

$$
\sigma_{0}=\Delta \alpha * \Delta E * \Delta T
$$

with

$$
\begin{aligned}
& \Delta \alpha=\alpha_{1}\left(1+v_{1}\right)-\alpha_{2}\left(1+v_{2}\right) . \\
& \Delta E=\left[\frac{1}{E_{1}^{*}}-\frac{1}{E_{2}{ }^{*}}\right]^{-1} \\
& E^{*}=\frac{E}{v(1+v)} .
\end{aligned}
$$

where, $\Delta T=T-T_{0}$ is the temperature difference, $T$ is the working temperature, and $T_{0}$ is the initial temperature. $E$ is the Young modulus, and $E^{*}$ is the effective modulus. $\alpha$ is the thermal expansion coefficient.

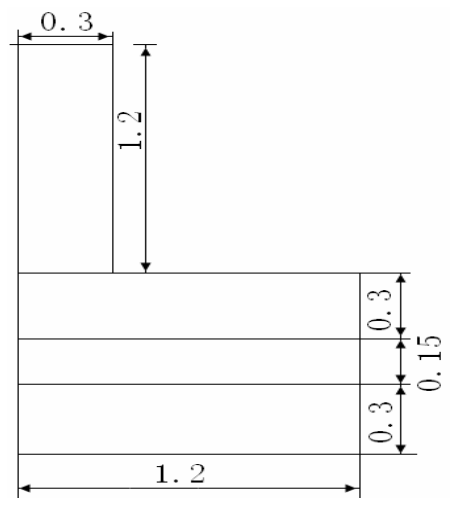

Fig. 2. Section of the Package Components

\section{Analysis of the QFP Welded Joints}

The welded pin joint in a QFP structure is shown in Fig.2. The upper layer is the pin, the bottom layer is the PCB, and the medium layer is the solder. The detailed dimension of these components is shown in Fig.2. The material properties for the pin, PCB and solder are listed in Table.1.The finite element model of the welded joint is given in Fig.3. In order to guarantee the calculation accuracy, the meshes near the interface edges are refined systematically. During the compu-tation and analysis, the finite element model must be based on the following assumptions(Jamil Wakil,and Paul S Ho 2007)[12]: 
1) Temperature for the thermal analysis dropped from $183^{\circ} \mathrm{C}$ to an ambient environmental temperature of $20^{\circ} \mathrm{C}$, since the QFP package is in a stress free state over $183^{\circ} \mathrm{C}$-the melting point temperature of the solder. Simplifications are made to facilitate prediction of junction temperatures and therm-mechanical stresses.

2) Thermal cycling was not considered, and the temperature dropped from $183^{\circ} \mathrm{C}$ to $20^{\circ} \mathrm{C}$ in 90 minutes.

3) The temperature condition was applied simul-taneously to the entire package.

4) A horizontal line on the bottom substrate surface was fixed along the Y-direction to constrain free body motion. The upper horizontal line of the pin was constrained using the same pattern. These were the boundary conditions of the analysis model.

5) All component interfaces were assumed to adhere perfectly and the material properties do not change with the temperature.

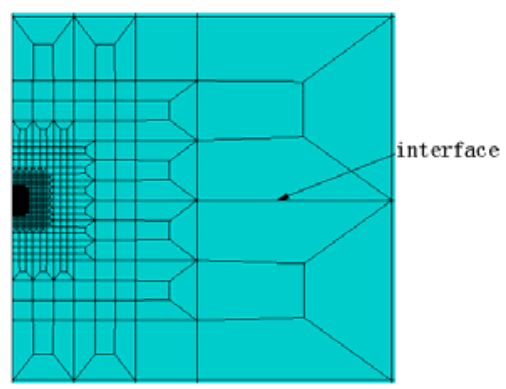

Fig. 3. Finite element model of the QFP

Table 1. Thermodynamics parameters of the materials in the model

\begin{tabular}{|c|l|l|l|c|c|}
\hline Material & E (Mpa) & $\nu$ & $\rho$ & $\alpha$ & c \\
\hline $\mathrm{Cu}$ & 120658 & 0.345 & 8900 & $17 \mathrm{e}-5$ & 385 \\
\hline $\mathrm{Pb} 40 \mathrm{Sn} 60$ & 34590 & 0.38 & 8400 & $21 \mathrm{e}-5$ & 176 \\
\hline $\mathrm{FR} 4$ & 22000 & 0.28 & 1800 & $18 \mathrm{e}-5$ & 930 \\
\hline
\end{tabular}

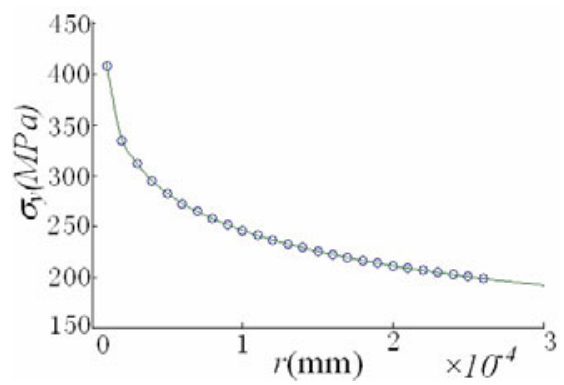

Fig. 4. Distribution of the peeling stress along the interface 


\section{Result and Discussion}

Fig.4 presented the peeling stress distribution along the interface of pin and PCB resulted from the cooling process. It was obvious that strong stress singularities existed at the point $\mathrm{o}$, and the interface delamination may initiate from here.

Once the peeling stress $\sigma_{y}$ and the constant stress term $\sigma_{0}$ are obtained, according to Eq. (1), the $\lambda$ and $\mathrm{K}$ can be obtained from the following plots:

$$
\lg \left(\sigma_{\theta}{ }^{F E}-\sigma_{0}\right)=\lg K-\lambda \lg (r / L) .
$$

where $\sigma_{\theta}{ }^{F E}$ is the stress value calculated by the finite element method. Fig. 5 shows an example of these plots. It was found that the slope of the straight line through the data points in the range $-4<\lg (r / L)<-3.2$ was in good agreement with the analytically calculated $\lambda$ in Munz and Yang [4]. The y-intercept of the fitting curve represents the $\operatorname{lgK}$ and the slope of the fitting curve represents the $-\lambda$.

Fig. 6 shows the effect of $\alpha_{2}$ on the stress singularity order $\lambda$. It was found that the stress singularity order $\lambda$ was decreased with the increasing of $\alpha_{2}$. Fig.7 shows the effect of $\alpha_{2}$ on $K 1 / \Delta T$. It can be seen that the $K 1 / \Delta T$ increases with the increasing of $\alpha 2$. The stress intensity factor $K 1$ is a very important parameter for crack initiation, i.e., when the $K 1$ reaches an ultimate value, the interface failure will occur.

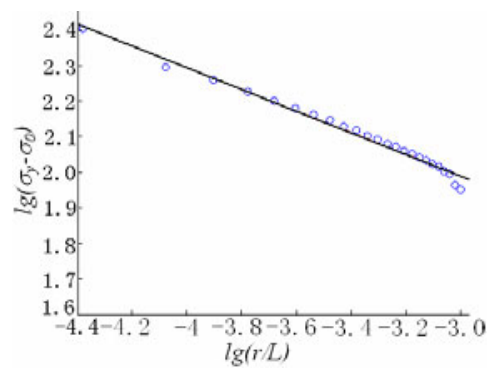

Fig. 5. Relation between $\lg \left(\sigma_{\mathrm{y}}-\sigma_{0}\right)$ and $\lg (r / L)$

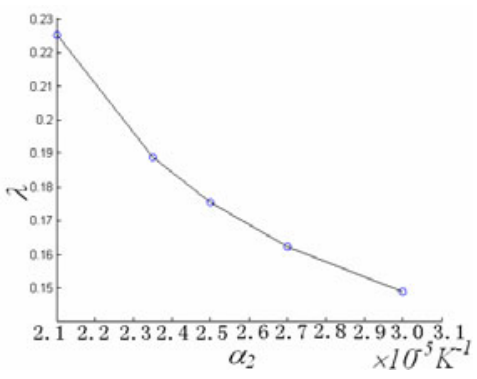

Fig. 6. $\lambda$ 's variation against different $\alpha_{2}$ 


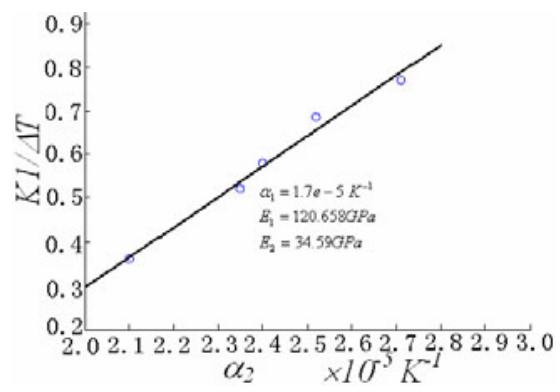

Fig. 7. $K 1 / \Delta T$ varied against different $\alpha_{2}$

Fig. 8 showed the effect of changing $\alpha 2$ on the $\mathrm{K} 1 / \sigma_{0}$. The result was that larger $\alpha_{2}$ leads to larger K1/ $\sigma_{0}$. Fig.9 showed the effect of the poisson's ratio on the term $\mathrm{K} 1 / \Delta \mathrm{T}$ including the stress intensity factor. In order to study the effect of the mismatch of thermal and mechanical properties of the bonded materials on the stress distribution, the FE plot of $\sigma_{\mathrm{y}} / \Delta \mathrm{T}$ versus $\mathrm{k}\left(=\frac{E_{1}{ }^{*}-E_{2}{ }^{*}}{E_{1}{ }^{*}+E_{2}{ }^{*}}\right)$ was given in Fig.10. It can be seen that $\sigma_{\mathrm{y}} / \Delta \mathrm{T}$ decreases with the decreasing of $\mathrm{k}$. In some cases (e.g. $\left.\mathrm{k}<0.2\right)$, the compressive stresses will appear.

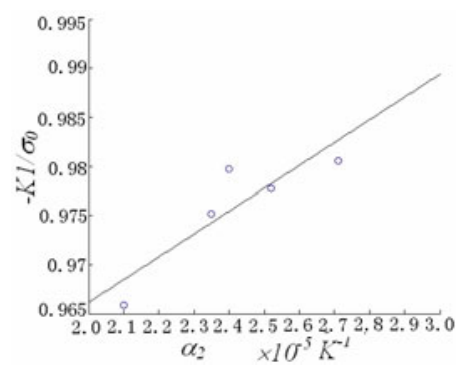

Fig. 8. $-K 1 / \sigma_{0}$ varied against different $\alpha_{2}$

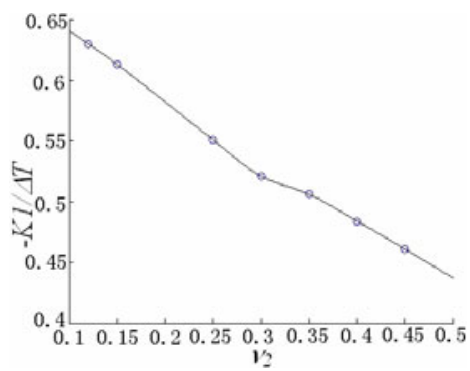

Fig. 9. $-K 1 / \Delta T$ varied against different $v_{2}$ 


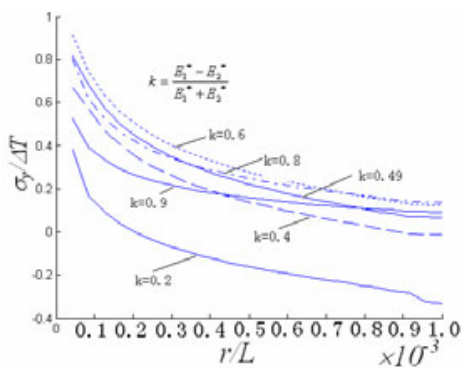

Fig. 10. $\sigma_{Y} / \Delta_{T}$ varied against $R / L$ with different $K$

In Fig.11, the effect of thickness of the solder layer on the peeling stress $\sigma y / \Delta T$ was considered. In the analysis, the thermal and mechanical properties of the materials were constants. It was easy to see that the peeling stress $\sigma \mathrm{y} / \Delta \mathrm{T}$ will increase with the increasing of the solder layer's thickness. Therefore, thinner solder layer is recommended on the premise of enough bonding strength.

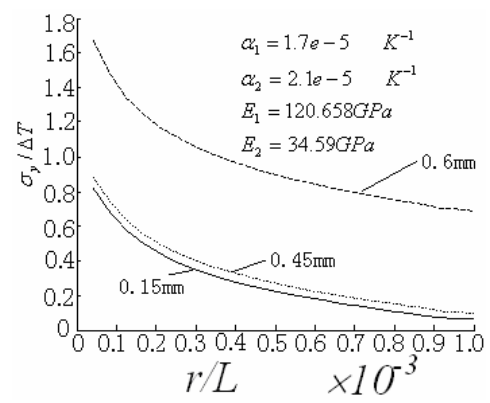

Fig. 11. $\sigma \mathrm{y} / \Delta \mathrm{T}$ varied against different solder thickness

\section{Conclusions}

The thermal stress distribution at the interface between the chip and PCB was affected by the material's properties and the structure's dimension. The peeling stress's distribution is also dependent on the solder layer's thickness. The effects of $\alpha_{2}$ on $K 1$ and $\lambda$ are different, and the stress intensity factor $K 1$ is very important to predict the interface failure occurrence. The peeling stresses are directly proportional to the $r^{-\lambda}$ and the stress values near the free edges (when $r$ approaches to zero) are very rigorous theoretically. It is in accordance with the results of the finite element analysis. The analysis of thermal stresses along the interface is very important for guiding the good design of packaging structures.

Acknowledgements. The authors acknowledge the support of National Natural Science Foundation of China through grant No.10362002 and No.10662004, the Jiangxi 
Provincial Natural Science Foundation of China through grant No. 2007GZW0862 and the Scientific Research Project of Jiangxi Provincial Department of Education grant NO. GJJ10444.

\section{References}

1. Xu, A.Q., Nied, H.F.: Finite Element Analysis of Stress Singularities in Attached Flip Chip Packages. ASME. Journal of Electronic Packaging, 301-305 (2000)

2. Kang, Y.: The thermal stress analysis of the interfacial edge in dissimilar structure and the effects of geometric shape. Transactions of tianjin university 2(2) (1996)

3. Suhir, E.: Thermally induced interfacial stresses in elongated bimaterial plates. Appl. Mech. Rev. 42(11), s253 (1989)

4. Munz, D., Yang, Y.Y.: Stress singularities at the interface in bonded dissimilar materials under mechanical and thermal loading. Journal of Applied Mechanics 59(4), 857-861 (1992)

5. Kang, Y.L., Learmann, K.H., Jia, Y.Q.: Experimental analysis for bonded bimaterial beam under bending load. Measurement 15(2), 85-90 (1995)

6. Chue, C.-h., Liu, C.-I.: A general solution on stress singularities in an anisotropic wedge. International Journal of Solids and Structures 38, 6889-6906 (2001)

7. Basaran, C., Ye, H.: Failure Modes of Flip Chip Solder Joints Under High Electric Current Density. Journal of Electronic Packaging 127, 157-163 (2005)

8. Park, J.-W., Mendez, P.F., Eagar, T.W.: Strain energy distribution in ceramic-to-metal joints. Acta Materialia 50, 883-899 (2002)

9. Jiang, C-s, Xie, K-j.: Interfacial Thermal Stress Analysis in Packaging. Electronic \& Packaging (2006)

10. Cho, S., Choi, J.: Study on the Behavior Characteristics of Solder Balls for FCBGA Package. Met. Mater. Int. 15(2), 299-305 (2009)

11. Hu, J.M.: Interfacial stress singularity analysis: A case study for plastic encapsulated IC packages. Application of Fracture Mechanics in Electronic Packaging and Materials, AS/VIE EEP-11/ MD-64, 13-21 (1995)

12. Wakil, J., Ho, P.S.: Effect of non-uniform temperature on thermal modeling and strain distribution in Interlayer. International Journal of Solids and Structures 44, 6232-6238 (2007)

13. Ioka, S., Masuda, K., et al.: Singular stress field near the edge of interface of bonded dissimilar materials with an electronic packaging. In: Electronic Components and Technology Conference, pp. 330-337 (1999) 